\title{
A Comparative Study of Levels of Total Leukocytes, Neutrophils and Lymphocytes in Blood of Brucellosis Affected, Vaccinated and Unvaccinated Healthy Cattle
}

\author{
Sugandha Raj ${ }^{1}$, Hari Mohan Saxena ${ }^{1 *}$ and S.T. Singh ${ }^{2}$ \\ ${ }^{1}$ Departments of Veterinary Microbiology, ${ }^{2}$ Department Animal Genetics and Breeding, Guru \\ Angad Dev Veterinary and Animal Sciences University, Ludhiana, Punjab, India \\ *Corresponding author
}

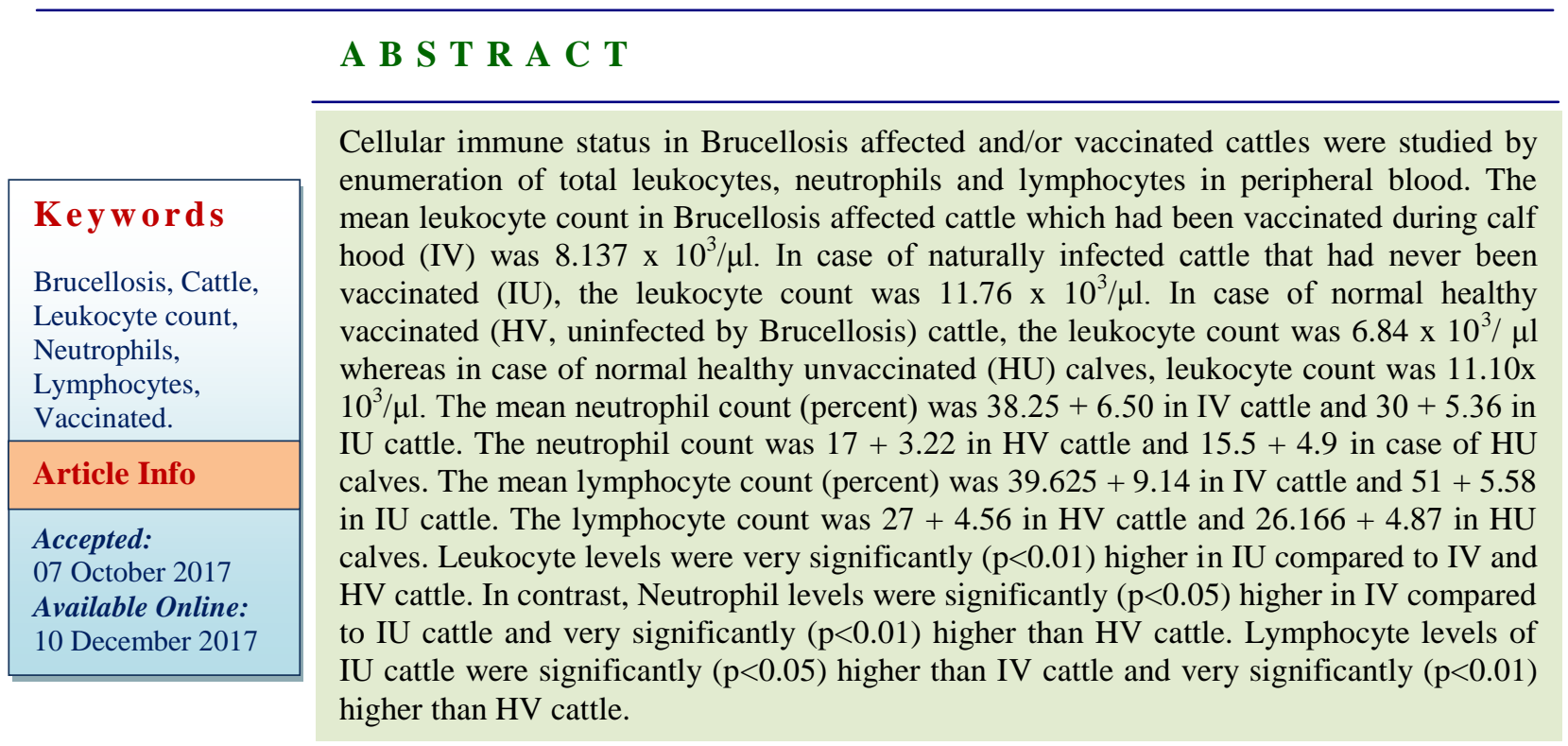

\section{Introduction}

Brucellosis is a zoonotic disease of domestic animals, wild animals and humans. About 500,000 cases of human Brucellosis are estimated to occur worldwide every year. Bovine Brucellosis is endemic in all states of India. The occurrence of Brucellosis in India is reportedly to the extent of $10 \%$ in the marginal herds and $50 \%$ in organized farms. The economic impact of the disease was estimated to be Rs. 500 crores annually (Jain et al., 2013; Pandeya et al., 2013). It causes heavy economic loss to the animal industry through delayed conception, late-term abortions and retention of placenta and temporary or permanent infertility (Kollannur et al., 2007) in females and orchitis and epididymitis in males, with excretion of organisms in semen, uterine discharges, and in milk (Godfroid et al., 2013). The immune response induced by Brucella is complex, involving both humoral and cellular effectors (Poester et al., 2013). Mediators of humoral immunity such as antibody, complement and lysozyme can remove microorganisms by 
opsonization and bactericidal actions. Cellular components of the immune system, including macrophages, dendritic cells, neutrophils and innate $\mathrm{T}$ cells, have major roles in innate immunity. However, little is known about the immune cell profile after vaccination and during disease in cattle.

Although it is well known that cell mediated immunity plays an important role in elimination of intracellular pathogens like Brucella, the S19 vaccine currently in use for bovine Brucellosis is the one which promotes antibody rather than cellular response. It is no wonder that a substantial portion of vaccinated animals succumb to Brucellosis and keep on spreading infection to other healthy animals also on becoming carrier.

It would be useful to compare the status of cell mediated immunity in naturally infected animals with those of vaccinated animals. We have therefore undertaken the present study to assess the levels of cells involved in mediating immunity against Brucella organisms. We studied the total blood leukocytes and neutrophils mediating innate immunity and lymphocytes responsible for adaptive immunity in Brucellosis affected vaccinated or unvaccinated cattle as well as healthy vaccinated cattle. The valuable insight yielded by this study could be of help in devising better strategies for the control of bovine Brucellosis.

\section{Materials and Methods}

Permission of the Institutional Animal Ethics Committee (IAEC) was obtained and IAEC guidelines were followed in all animal experiments.

\section{Collection of blood}

Blood samples were collected from naturally infected clinical cases of Brucellosis in cattle which had never been vaccinated in villages in and around Ludhiana district and from Brucellosis affected cattle which had been vaccinated during calf hood, normal healthy (uninfected) vaccinated cattle and healthy unvaccinated calves from a dairy farm in Ludhiana.

\section{Rose Bengal Plate Test (RBPT)}

RBPT performed as per the method of Morgan et al., (1978) was used as a screening test to identify Brucellosis affected animals. Equal volumes $(25 \mu 1$ each) of RBPT colored antigen (Punjab Veterinary Vaccine Institute, Ludhiana) and test serum were mixed on a clean glass slide.

The slide was observed till $3 \mathrm{~min}$ for formation of clumps.

The formation of clumps indicated a positive reaction (agglutination) while the absence of clear clumps was considered as a negative reaction.

\section{Total leucocyte count and differential leukocyte count in blood}

The Total Leukocyte Count (TLC) of the blood samples collected from cattle was determined by automatic Hematology Analyzer. Differential Leukocyte Count (DLC) was carried out manually by making blood smears on glass slides, Leishman's staining and examining under microscope.

\section{Statistical analysis}

Data pertaining to TLC and DLC in peripheral blood were statistically analyzed by ANOVA.

\section{Results and Discussion}

We studied cellular immune status in Brucellosis affected or vaccinated cattle by enumeration of total blood leukocytes, 
neutrophils and lymphocytes in the peripheral blood of cattle.

\section{Total leukocyte levels}

The mean leukocyte count (x $\left.10^{3} / \mu \mathrm{L}\right)$ in Brucellosis affected cattle which had been vaccinated during calf hood (IV) was 8.137 (Table 1). In case of naturally infected cattle that had never been vaccinated (IU), the leukocyte count was 11.76 (Table 2). In case of healthy vaccinated cattle uninfected by Brucellosis (HV), leukocyte count was 6.84 (Table 3) whereas in case of normal healthy unvaccinated (HU) calves, leukocyte count was 11.10 (Table 4, Fig. 1). The leukocyte count of Brucellosis infected unvaccinated cattle was very significantly $(p<0.01)$ higher than that of Brucellosis affected vaccinated cattle. The leukocyte count of healthy unvaccinated calves was very significantly $(p<0.01)$ higher than that of Brucellosis affected vaccinated cattle. The leukocyte count of unvaccinated infected cattle was very significantly $(\mathrm{p}<0.01)$ higher than that of healthy vaccinated cattle. The leukocyte count of healthy unvaccinated calves was very significantly $(p<0.01)$ higher than that of healthy vaccinated cattle. The difference between leukocyte counts of Brucellosis affected vaccinated cattle and healthy vaccinated cattle as well as between unvaccinated infected cattle and healthy unvaccinated calves was non-significant.

\section{Neutrophil levels}

The mean neutrophil count (percent) in Brucellosis affected cattle which had been vaccinated during calf hood was $38.25+6.50$ (Table 1). In case of naturally infected cattle that had never been vaccinated, the neutrophil count was $30+5.36$ (Table 2). In case of healthy vaccinated cattle uninfected by
Brucellosis the neutrophil count was $17+$ 3.22 (Table 3) whereas in case of healthy unvaccinated calves, neutrophil count was $15.5+4.9$ (Table 4, Fig. 2).

The Neutrophil count of Brucellosis affected vaccinated cattle was significantly $(p<0.05)$ higher than that of Brucellosis infected unvaccinated cattle and very significantly $(p<0.01)$ higher than that of healthy vaccinated cattle and healthy unvaccinated calves. The Neutrophil count of unvaccinated infected cattle was very significantly $(p<0.01)$ higher than that of healthy vaccinated cattle and healthy unvaccinated calves. The difference between the mean Neutrophil counts of healthy vaccinated cattle and healthy unvaccinated calves was nonsignificant.

\section{Lymphocyte levels}

The mean lymphocyte count (percent) in Brucellosis affected cattle which had been vaccinated during calf hood was $39.625+$ 9.14 (Table 1). In case of naturally infected cattle that had never been vaccinated, the lymphocyte count was $51+5.58$ (Table 2 ). In case of healthy (uninfected by Brucellosis) vaccinated cattle, lymphocyte count was $27+$ 4.56 (Table 3) whereas in case of healthy unvaccinated calves, lymphocyte count was $26.166+4.87$ (Table 4, Fig. 2). The Lymphocyte count of Brucellosis affected vaccinated cattle was significantly $(p<0.05)$ lower than that of unvaccinated infected cattle, but higher than that of healthy vaccinated cattle and healthy unvaccinated calves. The Lymphocyte count of unvaccinated infected cattle was very significantly $(p<0.01)$ higher than that of healthy vaccinated cattle and healthy unvaccinated calves. 
Table.1 Levels of leukocyte subsets in Brucellosis affected vaccinated cattle

\begin{tabular}{|c|c|c|c|c|}
\hline \multirow[t]{2}{*}{ S. No } & \multirow{2}{*}{$\begin{array}{l}\text { Animal } \\
\text { number }\end{array}$} & \multicolumn{3}{|c|}{ Cell counts } \\
\hline & & Total Leukocytes $\left(\times 10^{3} / \mu \mathrm{l}\right)$ & Neutrophils (\%) & Lymphocytes (\%) \\
\hline 1 & B1 & 9.3 & 36 & 54 \\
\hline 2 & B2 & 9.8 & 48 & 38 \\
\hline 3 & B3 & 8.10 & 37 & 33 \\
\hline 4 & B4 & 9.50 & 20 & 50 \\
\hline 5 & B5 & 8.00 & 42 & 67 \\
\hline 6 & B6 & 10.1 & 45 & 37 \\
\hline 7 & B7 & 8.36 & 38 & 40 \\
\hline 8 & B8 & 8.41 & 47 & 52 \\
\hline 9 & B9 & 7.6 & 30 & 31 \\
\hline 10 & $\mathrm{~B} 10$ & 7.1 & 44 & 41 \\
\hline 11 & B11 & 7.6 & 37 & 24 \\
\hline 12 & B12 & 7.92 & 43 & 35 \\
\hline 13 & B13 & 8.23 & 38 & 37 \\
\hline 14 & B14 & 8.26 & 36 & 39 \\
\hline 15 & B15 & 7.8 & 32 & 30 \\
\hline 16 & B16 & 9.8 & 46 & 40 \\
\hline 17 & B17 & 7.61 & 32 & 30 \\
\hline 18 & B18 & 9.1 & 41 & 31 \\
\hline 19 & B19 & 7.12 & 44 & 45 \\
\hline 20 & B20 & 5.99 & 35 & 40 \\
\hline 21 & B21 & 7.02 & 35 & 37 \\
\hline 22 & $\mathrm{~B} 22$ & 7.04 & 44 & 38 \\
\hline 23 & $\mathrm{~B} 23$ & 8.32 & 35 & 42 \\
\hline 24 & B24 & 7.21 & 33 & 40 \\
\hline \multicolumn{2}{|c|}{ Mean + SD } & $8.137 \pm 1.03$ & $38.25 \pm 6.50$ & $39.625 \pm 9.14$ \\
\hline
\end{tabular}

Table.2 Total leukocyte count and differential leukocyte count in Brucellosis affected unvaccinated cattle

\begin{tabular}{|c|c|c|c|}
\hline Animal no. & TLC $(\mathbf{x} \mathbf{1 0} / \boldsymbol{\mu l})$ & Neutrophils $\mathbf{( \% )}$ & Lymphocytes (\%) \\
\hline A I & 12.73 & 36 & 58 \\
\hline A II & 11.90 & 22 & 54 \\
\hline A III & 11.46 & 28 & 49 \\
\hline A IV & 11.28 & 36 & 42 \\
\hline A V & 11.17 & 28 & 49 \\
\hline A VI & 12.04 & 30 & 54 \\
\hline Mean + SD & $\mathbf{1 1 . 7 6} \pm \mathbf{0 . 5 8}$ & $\mathbf{3 0} \pm \mathbf{5 . 3 6}$ & $\mathbf{5 1} \pm \mathbf{5 . 5 8}$ \\
\hline
\end{tabular}


Table.3 Total leukocyte count and differential leukocyte count in healthy vaccinated cattle

\begin{tabular}{|l|c|c|c|}
\hline Animal number & TLC $(\mathbf{x 1 0} \boldsymbol{3} / \boldsymbol{\mu l})$ & Neutrophils (\%) & Lymphocytes (\%) \\
\hline C1 & 7.93 & 18 & 30 \\
\hline C2 & 6.14 & 16 & 28 \\
\hline C3 & 7.01 & 12 & 33 \\
\hline C4 & 6.89 & 17 & 27 \\
\hline C5 & 6.04 & 17 & 24 \\
\hline C6 & 7.08 & 22 & 20 \\
\hline Mean \pm SD & $\mathbf{6 . 8 4} \pm \mathbf{0 . 6 9}$ & $\mathbf{1 7} \pm \mathbf{3 . 2 2}$ & $\mathbf{2 7} \pm \mathbf{4 . 5 6}$ \\
\hline
\end{tabular}

Table.4 Total leukocyte count and differential leukocyte count in healthy unvaccinated calves

\begin{tabular}{|l|c|c|c|}
\hline Animal no. & Total Leukocytes $\mathbf{( x 1 0} \boldsymbol{3} / \boldsymbol{\mu l})$ & Neutrophils (\%) & Lymphocytes (\%) \\
\hline D1 & 10.70 & 14 & 27 \\
\hline D2 & 9.87 & 18 & 27 \\
\hline D3 & 11.98 & 12 & 32 \\
\hline D4 & 12.01 & 15 & 30 \\
\hline D5 & 10.27 & 10 & 22 \\
\hline D6 & 11.82 & 24 & 19 \\
\hline Mean $\mathbf{\text { SD }}$ & $\mathbf{1 1 . 1 0 8} \underline{\mathbf{0 . 9 4}}$ & $\mathbf{1 5 . 5} \pm \mathbf{4 . 9}$ & $\mathbf{2 6 . 1 6 6} \pm \mathbf{4 . 8 7}$ \\
\hline
\end{tabular}

Fig.1 Total leukocyte counts in Brucellosis infected and vaccinated cattle

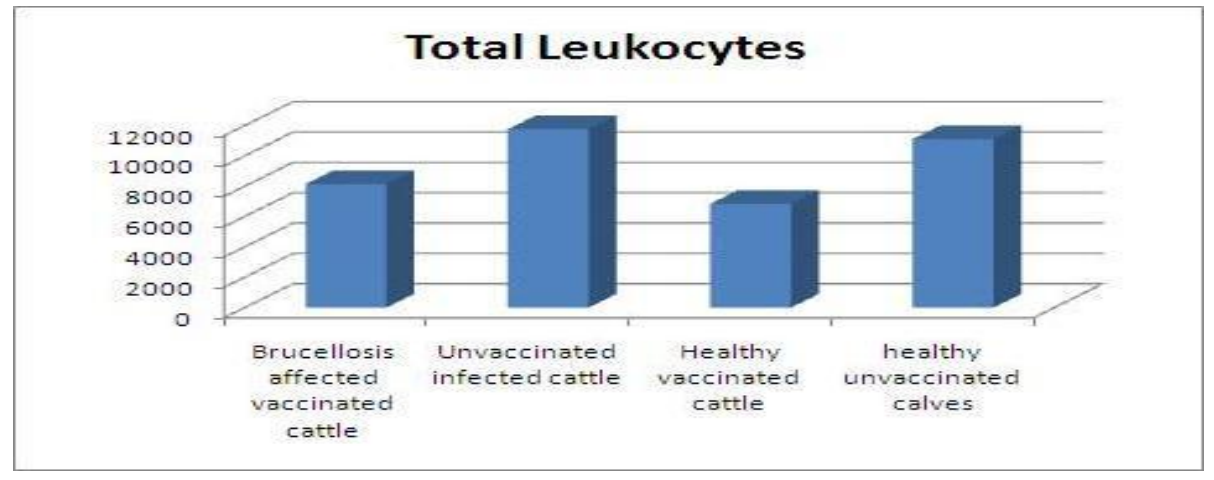

Fig.2 Levels of neutrophils and lymphocytes in Brucellosis infected and vaccinated cattle

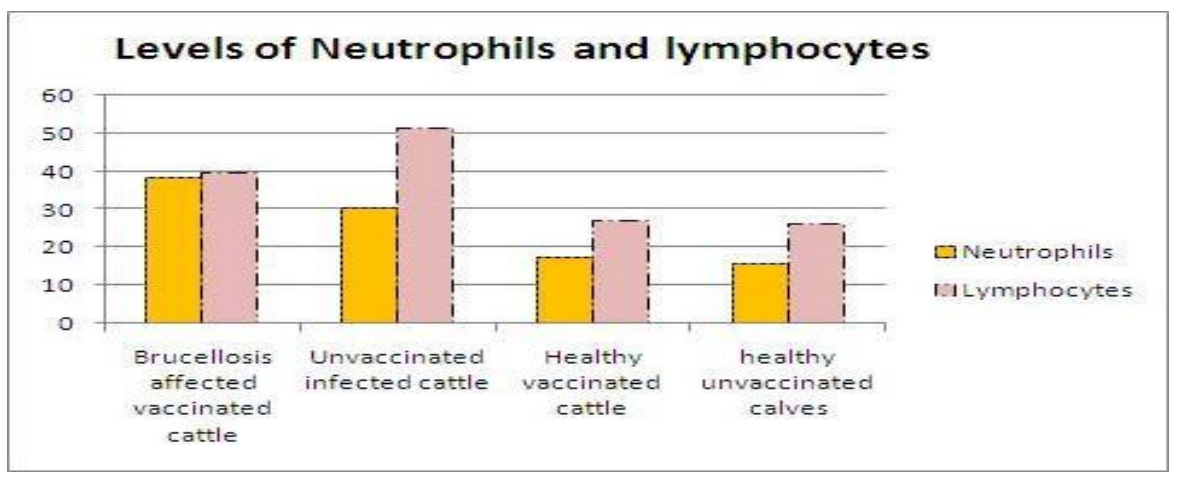


The difference between mean values of healthy vaccinated cattle and healthy unvaccinated calves was nonsignificant.

It was interesting to find that the leukocyte counts were very significantly higher in naturally infected cattle that had never been vaccinated compared to Brucellosis affected cattle vaccinated during calfhood, as well as in healthy vaccinated cattle. Conspicuously, leukocyte count in healthy unvaccinated calves was comparable to the unvaccinated infected cattle and the difference between the two was non-significant. A possible reason for the decreased leukocyte count in vaccinated cattle affected with Brucellosis could be the mitigation of pathogenesis of infection due to the earlier priming of immune system by vaccination. Higher levels in healthy unvaccinated calves may suggest a suppressive role of vaccination on leukocyte proliferation.

Immunohematological data is helpful in charting the disease process, diagnosis and prognosis and may help in understanding the pathophysiology of infection in cattle.

Sikder et al., (2012) carried out a hematological study to determine the variations in blood parameters of Brucellosis seropositive commercial dairy cattle. No significant $(p<0.05)$ variations were found in TLC and DLC between the Brucellosis positive and negative group. However, slightly increased values of TLC and monocytes were recorded in the positive group. In addition, a little decline in the values of neutrophil was found in the same group. The values of lymphocytes remained unchanged. They concluded that Brucella organisms are not responsible for a significant change in the hematological values. However, as evident from our results, overall suppression of cellular immune responses prevailed in Brucellosis infected animals.
Cell mediated immunity involves activation of the bactericidal mechanisms of antigen presenting cells (e.g., macrophages and dendritic cells) and the subsequent expansion of antigen specific CD4 and CD8 T cells. Brucella antigens induce the production of type 1 helper $\mathrm{T}$ cell $\left(\mathrm{T}_{\mathrm{H}} 1\right)$ cytokines and an adequate $T_{H} 1$ immune response is critical for the clearance of Brucella infection (Baldwin and Goenka, 2006). The L7/L12 ribosomal proteins are important in stimulating cellmediated responses (Oliveira and Splitter, 1994).

Since Brucellosis is a disease where cell mediated immunity is vital and vaccination with S19 is known to induce antibody response rather than cellular immune response, the finding of our study may have implications for devising a suitable strategy for control of Brucellosis.

Leukocyte levels were very significantly $(\mathrm{p}<0.01)$ higher in IU compared to IV and HV cattle. Neutrophil levels were significantly $(\mathrm{p}<0.05)$ higher in IV compared to IU cattle and very significantly $(\mathrm{p}<0.01)$ higher than HV cattle. Lymphocyte levels of IU cattle were significantly $(p<0.05)$ higher than IV and very significantly $(\mathrm{p}<0.01)$ higher than HV cattle.

\section{Conflict of interest}

The authors declare that there is no conflict of interest.

\section{Acknowledgements}

The study was funded by a grant under RKVY scheme (PI: H M Saxena).

\section{References}

Baldwin C L, and Goenka R (2006) Host immune responses to the intracellular 
bacterium Brucella: does the bacterium instruct the host to facilitate chronic infection? Critical Reiews in Immunology 26: 407-442.

Godfroid J, Garin-Bastuji B, Saegerman C and Blasco J M. 2013. Brucellosis in terrestrial wildlife. Revue Scientifique et Technique de l'Office International des Epizooties 32(1): 27-42.

Jain, U., Bisht, B., Sahzad, P. and Dwivedi, K. (2013) Outbreak of Brucellosis in buffaloes aborted in a village Mahuan, district Mainpuri, UP, India - A case report. Vet. World, 6(1): 51-52.

Kollannur, J.D., Rathore, R. and Chauhan, R.S. (2007) Epidemiology and economics of Brucellosis in animals and its zoonotic significance. Proceedings of XIII International Congress in Animal Hygiene. International Society for Animal Hygiene. p466 - 468.

Morgan W J, Mackinnon D T, Gill K P W, Gower S G M and Norris P I W. 1978. Brucellosis diagnosis: Standard Laboratory Techniques Report Series no. 1, Weybridge, England.

Oliveira, S.C. and Splitter, G.A. (1994) Subcloning and expression of Brucella abortus L7/L12 ribosomal gene and Tlymphocyte recognition of the recombinant protein. Infect. Immun., 62: 5201-5204.

Pandeya, Y.R., Joshi, D.D., Dhakal, S., Ghimire, L., Mahato, B.R., Chaulagain, S., Satyal, R.C. and Sah, S.K. (2013) Seroprevalence of Brucellosis in different animal species of Kailali district Nepal. International Journal of Infection and Microbiology 2 (1): 2225.

Poester F P, Nielsen K and Samartino L E. 2010. Diagnosis of brucellosis. The Open Veterinary Science Journal 4: 4660.

Sikder S, Rahman SSM, Alim MA and Das S (2012) Hematological Variations in Brucella abortus Antibody Positive Cross-bred Cattle at Chittagong, Bangladesh. YYU Veteriner Fakultesi Dergisi 23 (3), 125 - 128.

Splitter G., Oliveira S., Carey M., Miller C., Ko J. and Covert J. (1996) T lymphocyte mediated protection against facultative intracellular bacteria. Vet. Immunol. Immunopathol. 54:309-319.

\section{How to cite this article:}

Sugandha Raj, Hari Mohan Saxena and Singh, S.T. 2017. A Comparative Study of Levels of Total Leukocytes, Neutrophils and Lymphocytes in Blood of Brucellosis Affected, Vaccinated and Unvaccinated Healthy Cattle. Int.J.Curr.Microbiol.App.Sci. 6(12): 828-834. doi: https://doi.org/10.20546/ijcmas.2017.612.088 